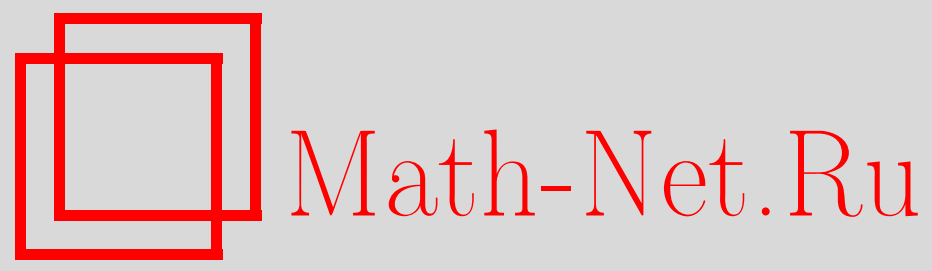

В. И. Афанасьев, Функциональная предельная теорема для критического ветвящегося процесса в случайной среде, Дискрет. матем., 2001, том 13, выпуск 4, 73-91

DOI: https://doi.org/10.4213/dm300

Использование Общероссийского математического портала Math-Net.Ru подразумевает, что вы прочитали и согласны с пользовательским соглашением http://www.mathnet.ru/rus/agreement

Параметры загрузки :

IP : 107.22 .136 .117

26 апреля 2023 г., 13:44:11

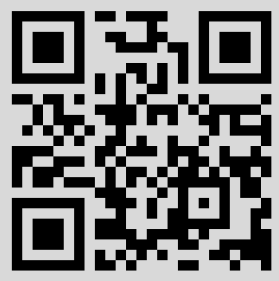




\section{Функциональная предельная теорема для критического ветвящегося процесса в случайной среде}

(c) 2001 г. В. И. Афанасьев

Пусть $\left\{\xi_{n}\right\}-$ критический ветвящийся процесс в случайной среде, $m_{n}-$ математическое ожидание $\xi_{n}$, вычисленное при условии, что случайная среда фиксирована. Доказана теорема о сходимости последовательности случайных процессов $\left\{\xi_{[n t]} / m_{[n t]}, t \in(0,1] \mid \xi_{n}>0\right\}$ при $n \rightarrow \infty$ по распределению в соответствующем функциональном пространстве. Эта теорема обобщает предшествующий результат автора, доказанный в предположении, что производящие функции числа непосредственных потомков дробно-линейны.

1. Пусть $\left\{\xi_{n}, n \in \mathbf{N}_{0}\right\}$ - ветвящийся процесс в случайной среде $\left\{\pi_{n}, n \in \mathbf{N}_{0}\right\}$, где

$$
\begin{aligned}
\pi_{n}=\left\{\pi_{n}(0), \pi_{n}(1), \pi_{n}(2), \ldots\right\}, \quad \pi_{n}(i) \geqslant 0, \quad i=1,2, \ldots, & \\
& \sum_{i=0}^{\infty} \pi_{n}(i)=1, \quad n \in \mathbf{N}_{0}=\{0,1,2, \ldots\},
\end{aligned}
$$

причем последовательности $\pi_{n}$ независимы и одинаково распределены при разных $n$. По определению это означает, что случайные величины $\xi_{n}$ являются неотрицательными целочисленными и

$$
\xi_{0}=1, \quad \mathbf{M}\left(s^{\xi_{n+1}} \mid \xi_{0}, \xi_{1}, \ldots, \xi_{n}, \pi_{0}, \pi_{1}, \ldots, \pi_{n}\right)=\left(f_{n}(s)\right)^{\xi_{n}}
$$

где

$$
f_{n}(s)=\sum_{i=0}^{\infty} \pi_{n}(i) s^{i}, \quad s \in[-1,1], \quad n \in \mathbf{N}_{0}
$$

Положим

$$
X_{i}=\ln f_{i-1}^{\prime}(1), \quad \eta_{i}=f_{i-1}^{\prime \prime}(1) /\left(2 f_{i-1}^{\prime}(1)^{2}\right), \quad i \in \mathbf{N} .
$$

Будем предполагать, что процесс $\left\{\xi_{n}\right\}$ является критическим, то есть $\mathbf{M} X_{1}=0$, и выполнены условия

(a) конечны $\mathbf{M} X_{1}^{2}, \mathbf{M} \eta_{1}, \mathbf{M} X_{1} \eta_{1}$, причем $\mathbf{M} X_{1}^{2}=\sigma^{2}>0$,

(b) $M \exp \left(-X_{1}\right)<\infty$. 
Пусть $T=\inf \left\{n: \xi_{n}=0\right\}-$ момент вырождения $\left\{\xi_{n}\right\}$. Положим

$$
m_{n}=\mathbf{M}_{\pi} \xi_{n}
$$

(символы $\mathbf{M}_{\boldsymbol{\pi}}$ и $\mathbf{P}_{\boldsymbol{\pi}}$ означают математическое ожидание и вероятность, вычисленные при условии, что случайная среда $\left\{\pi_{n}\right\}$ фиксирована).

Цель настоящей работы - доказательство следующей функциональной предельной теоремы.

Теорема 1. Пусть $\left\{\xi_{n}\right\}$ - критический ветвящийся прочесс в случайной среде $\left\{\pi_{n}\right\}$ и въполненъ условия (a) $u(\mathrm{~b})$, тогда при $n \rightarrow \infty$

$$
\left\{\frac{\xi_{[n t]}}{m_{[n t]}}, \quad t \in(0,1] \mid T>n\right\} \stackrel{\mathscr{D}}{\longrightarrow} \mu,
$$

где $\mu$ - случайный процесс с неотрицателънъми постоянными реализациями на $(0,1]$, причем $\mathbf{P}(\mu>0)>0$, сходимость в (2) надо понимать как сходимость по распределению в пространстве $D[u, 1]$ с топологией Скорохода при яюбом фиксированном $u \in(0,1)$.

Указанная теорема обобщает результат автора [1], где помимо условий (a), (b) предполагается, что производящие функции $f_{n}(s), n \in \mathbf{N}_{0}$, являются дробно-линейными. Аналогичные теоремы для докритических ветвящихся процессов в случайной среде рассматриваются в работах автора $[2,3]$.

2. В данном разделе изучаются свойства так называемых сопровождающих случайных последовательностей. В силу того, что компоненты среды $\pi_{n}, n \in \mathbf{N}_{0}$, одинаково распределены и независимы, случайные величины $X_{1}, X_{2}, \ldots$ независимы и одинаково распределены. Рассмотрим сопровождающее случайное блуждание

$$
S_{0}=0, \quad S_{n}=\sum_{i=1}^{n} X_{i}, \quad n \in \mathbf{N} .
$$

Оно имеет нулевой снос, поскольку процесс $\left\{\xi_{n}\right\}$ является критическим. Пусть $\tau_{0}=0, \tau_{1}, \tau_{2}, \ldots$ - строгие нижние лестничные моменты этого блуждания, то есть

$$
\tau_{1}=\inf \left\{n: S_{n}<0\right\}, \quad \tau_{2}=\inf \left\{n>\tau_{1}: S_{n}<S_{\tau_{1}}\right\}, \ldots
$$

В работе М. В. Козлова [4] установлено, что если $\mathbf{M} X_{1}=0$ и $\mathbf{M} X_{1}^{2}<\infty$, то при любом фиксированном $j \in \mathbf{N}_{0}$ и $n \rightarrow \infty$, во-первых,

$$
\mathbf{P}\left(\tau_{j} \leqslant n<\tau_{j+1}\right) \sim \frac{c_{1}}{\sqrt{n}}
$$

где постоянная $c_{1}$ положительна и не зависит от $n$ и $j$, во-вторых, при любом фиксированном $\lambda \in(0,1)$

$$
\mathbf{P}\left(\lambda n<\tau_{j} \leqslant n<\tau_{j+1}\right)=o(1 / \sqrt{n}) .
$$

В [4] также доказано, что если $\mathbf{M} X_{1}=0, \mathbf{M} X_{1}^{2}<\infty$ и $\left\{\bar{Y}_{i}, i \in \mathbf{N}\right\}-$ последовательность случайных векторов одинаковой размерности $l$ такая, что случайные векторы $\left(X_{1}, \bar{Y}_{1}\right),\left(X_{2}, \bar{Y}_{2}\right), \ldots$ независимы и одинаково распределены, то

$$
\left\{\left(S_{i}, \bar{Y}_{i}\right), i \in \mathbf{N} \mid n<\tau_{1}\right\} \stackrel{D}{\longrightarrow}\left\{\left(S_{i}^{(1)}, \bar{Y}_{i}^{(1)}\right), i \in \mathbf{N}\right\}
$$


где справа в (5) стоит некоторая последовательность случайных векторов, причем $\left\{S_{i}^{(1)}, i \in \mathbf{N}\right\}$ является однородной марковской цепью, сходимость в (5) надо понимать как сходимость по распределению в $(l+1)$-й степени пространства $\mathbf{R}^{\infty} \mathrm{c}$ топологией покоординатной сходимости. Более того, если

$$
G(x, \Gamma)=\mathbf{P}\left(\bar{Y}_{1} \in \Gamma \mid X_{1}=x\right),
$$

где $x \in \mathbf{R}, \Gamma-$ борелевское множество из $\mathbf{R}^{l}$, то при любом фиксированном $k \in \mathbf{N}$ и любых борелевских множествах $D_{i} \in \mathbf{R}, \Gamma_{i} \in \mathbf{R}^{l}, i=1, \ldots, k$,

$$
\begin{aligned}
\lim _{n \rightarrow \infty} \mathbf{P}\left(S_{i} \in D_{i}, \bar{Y}_{i}\right. & \left.\in \Gamma_{i}, i=1, \ldots, k \mid n<\tau_{1}\right) \\
& =\int_{D_{1}} \ldots \int_{D_{k}} \prod_{i=1}^{k} G\left(x_{i}-x_{i-1}, \Gamma_{i}\right) d \mathbf{P}\left(S_{i}^{(1)} \leqslant x_{1}, i=1, \ldots, k\right) .
\end{aligned}
$$

Обобщим соотношения (5) и (6).

Лемма 1. Если $\mathrm{M} X_{1}=0 u \mathrm{M} X_{1}^{2}<\infty$, по при любом $j \in \mathbf{N}_{0}$

$$
\left\{\left(S_{i}, \bar{Y}_{i}\right), i \in \mathbf{N} \mid \tau_{j} \leqslant n<\tau_{j+1}\right\} \stackrel{\mathscr{D}}{\longrightarrow}\left\{\left(S_{i}^{(j)}, \bar{Y}_{i}^{(j)}\right), \in \mathbf{N}\right\}
$$

где справа стоит нехоторая последовательность случайных векторов, более того, при любом $k \in \mathbf{N}$ и любых борелевсхих множествах $D_{i} \in \mathbf{R}, \Gamma_{i} \in \mathbf{R}^{l}, i=1, \ldots, k$,

$$
\begin{aligned}
\lim _{n \rightarrow \infty} \mathbf{P}\left(S_{i} \in D_{i}, \bar{Y}_{i}\right. & \left.\in \Gamma_{i}, i=1, \ldots, k \mid \tau_{j} \leqslant n<\tau_{j+1}\right) \\
& =\int_{D_{1}} \ldots \int_{D_{k}} \prod_{i=1}^{k} G\left(x_{i}-x_{i-1}, \Gamma_{i}\right) d \mathbf{P}\left(S_{i}^{(j)} \leqslant x_{i}, i=1, \ldots, k\right) .
\end{aligned}
$$

Доказателъство. При $j=0$ доказываемая лемма справедлива ввиду соотношений (5), (6). Не ограничивая общности, можно считать, что предельная при $j=0$ последовательность случайных векторов $\left\{\left(S_{i}^{(1)}, \bar{Y}_{i}^{(1)}\right)\right\}$ задана на том же вероятностном пространстве, что и $\left\{\left(S_{i}, \bar{Y}_{i}\right)\right\}$, причем эти две последовательности независимы. Зафиксируем $j \in \mathbf{N}$. Очевидно, что при $n>k$

$$
\begin{aligned}
& \mathbf{P}\left(S_{i} \in D_{i}, \bar{Y}_{i} \in\right.\left.\Gamma_{i}, i=1, \ldots, k ; \tau_{j} \leqslant n<\tau_{j+1}\right) \\
&=\sum_{r=1}^{k} \mathbf{P}\left(S_{i} \in D_{i}, \bar{Y}_{i} \in \Gamma_{i}, i=1, \ldots, k, \tau_{j}=r, \tau_{j+1}>n\right) \\
& \quad+\mathbf{P}\left(S_{i} \in D_{i}, \bar{Y}_{i} \in \Gamma_{i}, i=1, \ldots, k, k<\tau_{j} \leqslant n<\tau_{j+1}\right) .
\end{aligned}
$$

Положим

$$
S_{i}^{\prime}=S_{\tau_{j}+i}-S_{\tau_{j}}, \quad \bar{Y}_{i}^{\prime}=\bar{Y}_{\tau_{j}+i}, \quad i \in \mathrm{N}_{0}, \quad \tau_{1}^{\prime}=\tau_{j+1}-\tau_{j} .
$$

Последовательность случайных векторов $\left\{\left(S_{i}^{\prime}, \bar{Y}_{i}^{\prime}\right)\right\}$ образует точную вероятностную копию последовательности $\left\{\left(S_{i}, \bar{Y}_{i}\right)\right\}$ и не зависит от $\tau_{j}, S_{1}, \bar{Y}_{1}, S_{2}, \bar{Y}_{2}, \ldots, S_{\tau_{j}}, \bar{Y}_{\tau_{j}}$. Заметим, что при $r \leqslant k$

$$
\begin{array}{r}
\mathbf{P}\left(S_{i} \in D_{i}, \bar{Y}_{i} \in \Gamma_{i}, i=1, \ldots, k, \tau_{j}=r, \tau_{j+1}>n\right)=\mathbf{P}\left(S_{i} \in D_{i}, \bar{Y}_{i} \in \Gamma_{i}, i=1, \ldots, r,\right. \\
\left.S_{r}+S_{p}^{\prime} \in D_{r+p}, \bar{Y}_{p}^{\prime} \in \Gamma_{r+p}, p=1, \ldots, k-r, \tau_{j}=r, \tau_{1}^{\prime}>n-r\right) .
\end{array}
$$


Отсюда, учитывая независимость $\left\{\left(S_{i}^{\prime}, \bar{Y}_{i}^{\prime}\right)\right\}$ от $\tau_{j}, S_{1}, \bar{Y}_{1}, \ldots, S_{\tau_{j}}, \bar{Y}_{\tau_{j}}$ и (3), (5), (6), получаем, что при $r \leqslant k$

$$
\begin{aligned}
\lim _{n \rightarrow \infty} \frac{\sqrt{n}}{c_{1}} \mathbf{P}\left(S_{i} \in D_{i},\right. & \left.\bar{Y}_{i} \in \Gamma_{i}, i=1, \ldots, k, \tau_{j}=r, \tau_{j+1}>n\right) \\
= & \mathbf{P}\left(S_{i} \in D_{i}, \bar{Y}_{i} \in \Gamma_{i}, i=1, \ldots, r, \tau_{j}=r\right. \\
& \left.S_{r}+S_{p}^{(1)} \in D_{r+p}, \bar{Y}_{p}^{(1)} \in \Gamma_{r+p}, p=1, \ldots, k-r\right) .
\end{aligned}
$$

Рассмотрим вероятности

$$
\begin{aligned}
P_{n} & =\mathbf{P}\left(S_{i} \in D_{i}, \bar{Y}_{i} \in \Gamma_{i}, i=1, \ldots, k, k<\tau_{j} \leqslant n<\tau_{j+1}\right), \\
P_{n}^{(1)} & =\mathbf{P}\left(S_{i} \in D_{i}, \bar{Y}_{i} \in \Gamma_{i}, i=1, \ldots, k, k<\tau_{j} \leqslant \lambda n, \tau_{j+1}>n\right), \\
P_{n}^{(2)} & =\mathbf{P}\left(S_{i} \in D_{i}, \bar{Y}_{i} \in \Gamma_{i}, i=1, \ldots, k, k<\tau_{j} \leqslant \lambda n, \tau_{1}^{\prime}>n\right), \\
P_{n}^{(3)} & =\mathbf{P}\left(S_{i} \in D_{i}, \bar{Y}_{i} \in \Gamma_{i}, i=1, \ldots, k, k<\tau_{j} \leqslant \lambda n, \tau_{1}^{\prime}>n(1-\lambda)\right),
\end{aligned}
$$

где $\lambda \in(0,1)$. Очевидно, что

$$
P_{n}^{(2)} \leqslant P_{n}^{(1)} \leqslant P_{n}^{(3)}
$$

Ввиду соотношения (3) и независимости $\left\{\left(S_{i}^{\prime}, \bar{Y}_{i}^{\prime}\right)\right\}$ от $\tau_{j}, S_{1}, \bar{Y}_{1}, \ldots, S_{\tau_{j}}, \bar{Y}_{\tau_{j}}$

$$
\begin{aligned}
& \lim _{n \rightarrow \infty} \frac{\sqrt{n}}{c_{1}} P_{n}^{(2)}=\mathbf{P}\left(S_{i} \in D_{i}, \bar{Y}_{i} \in \Gamma_{i}, i=1, \ldots, k, k<\tau_{j}\right), \\
& \lim _{n \rightarrow \infty} \frac{\sqrt{n}}{c_{1}} P_{n}^{(3)}=\frac{1}{\sqrt{1-\lambda}} \mathbf{P}\left(S_{i} \in D_{i}, \bar{Y}_{i} \in \Gamma_{i}, i=1, \ldots, k, k<\tau_{j}\right) .
\end{aligned}
$$

Следовательно,

$$
\begin{aligned}
\mathbf{P}\left(S_{i} \in D_{i}, \bar{Y}_{i} \in \Gamma_{i}, i=1, \ldots, k, k<\tau_{j}\right) \leqslant \varliminf_{n \rightarrow \infty} \frac{\sqrt{n}}{c_{1}} \mathbf{P}_{n}^{(1)} \leqslant \varlimsup_{n \rightarrow \infty} \frac{\sqrt{n}}{c_{1}} P_{n}^{(1)} \\
\leqslant \frac{1}{\sqrt{1-\lambda}} \mathbf{P}\left(S_{i} \in D_{i}, \bar{Y} \in \Gamma_{i}, i=1, \ldots, k ; k<\tau_{j}\right) .
\end{aligned}
$$

Очевидно, что

$$
\left\{\tau_{j} \leqslant n<\tau_{j+1}\right\}=\left\{\tau_{j} \leqslant \lambda n, \tau_{j+1}>n\right\}+\left\{\lambda n<\tau_{j} \leqslant n<\tau_{j+1}\right\} .
$$

Ввиду соотношений (4) и (12) верхние (нижние) пределы $\sqrt{n} P_{n}$ и $\sqrt{n} P_{n}^{(1)}$ при $n \rightarrow \infty$ совпадают, поэтому

$$
\begin{aligned}
\mathbf{P}\left(S_{i} \in D_{i}, \bar{Y}_{i} \in \Gamma_{i}, i=1, \ldots,\right. & \left.k, k<\tau_{j}\right) \leqslant \varliminf_{n \rightarrow \infty} \frac{\sqrt{n}}{c_{1}} P_{n} \leqslant \varlimsup_{n \rightarrow \infty} \frac{\sqrt{n}}{c_{1}} P_{n} \\
& \leqslant \frac{1}{\sqrt{1-\lambda}} \mathbf{P}\left(S_{i} \in D_{i}, \bar{Y}_{i} \in \Gamma_{i}, i=1, \ldots, k, k<\tau_{j}\right) .
\end{aligned}
$$

Так как вероятность $P_{n}$ не зависит от $\lambda$, переходя к пределу при $\lambda \rightarrow 0$, получаем, что

$$
\lim _{n \rightarrow \infty} \frac{\sqrt{n}}{c_{1}} P_{n}=\mathbf{P}\left(S_{i} \in D_{i}, \bar{Y}_{i} \in \Gamma_{i}, i=1, \ldots, k, k<\tau_{j}\right)
$$


Из соотношений $(3),(9),(10),(13)$ следует, что

$$
\begin{aligned}
& \lim _{n \rightarrow \infty} \mathbf{P}\left(S_{i} \in D_{i}, \bar{Y}_{i} \in \Gamma_{i}, i=1, \ldots, k \mid \tau_{j} \leqslant n<\tau_{j+1}\right) \\
& \begin{array}{c}
=\sum_{r=1}^{k} \mathbf{P}\left(S_{i} \in D_{i}, \bar{Y}_{i} \in \Gamma_{i}, i=1, \ldots, r, \tau_{j}=r, S_{r}+S_{p}^{(1)} \in D_{r+p},\right. \\
\left.\bar{Y}_{p}^{(1)} \in \Gamma_{r+p}, p=1, \ldots, k-r\right)
\end{array} \\
& +\mathbf{P}\left(S_{i} \in D_{i}, \bar{Y}_{i} \in \Gamma_{i}, i=1, \ldots, k, k<\tau_{j}\right) .
\end{aligned}
$$

Видно, что выражение в правой части (14) задает собственное распределение, поскольку при

$$
D_{1}=D_{2}=\ldots=D_{k}=\mathbf{R}, \quad \Gamma_{1}=\Gamma_{2}=\ldots=\Gamma_{k}=\mathbf{R}^{l}
$$

правая часть равна

$$
\sum_{r=1}^{k} \mathbf{P}\left(\tau_{j}=r\right)+\mathbf{P}\left(\tau_{j}>k\right)=1 .
$$

Итак, соотношение (7) доказано.

Из определения условной вероятности $G(x, \Gamma)$ следует, что

$$
\begin{aligned}
\mathbf{P}\left(S_{i} \in D_{i}, \bar{Y}_{i}\right. & \left.\in \Gamma_{i}, i=1, \ldots, k, k<\tau_{j}\right) \\
& =\int_{D_{1}} \ldots \int_{D_{k}} \prod_{i=1}^{k} G\left(x_{i}-x_{i-1}, \Gamma_{i}\right) d \mathbf{P}\left(S_{i} \leqslant x_{i}, i=1, \ldots, k, k<\tau_{j}\right) .
\end{aligned}
$$

Ввиду соотношения (6) и независимости последовательностей $\left\{\left(S_{i}^{(1)}, \bar{Y}_{i}^{(1)}\right)\right\}$ и $\left\{\left(S_{i}, \bar{Y}_{i}\right)\right\}$ при любом $r=1, \ldots, k$

$$
\begin{aligned}
\mathbf{P}\left(S_{i} \in D_{i}, \bar{Y}_{i} \in\right. & \left.\Gamma_{i}, i=1, \ldots, r, \tau_{j}=r, S_{r}+S_{p}^{(1)} \in D_{r+p}, \bar{Y}_{p}^{(1)} \in \Gamma_{r+p}, p=1, \ldots, k-r\right) \\
= & \mathbf{M}\left(\chi_{\left\{\left\{S_{i} \in D_{i}, \bar{Y}_{i} \in \Gamma_{i}, i=1, \ldots, r, \tau_{j}=r\right\}\right.}\right. \\
& \times \int_{D_{r+1}-S_{r}} \ldots \int_{D_{k}-S_{r}} \prod_{p=1}^{k-r} G\left(x_{r+p}-x_{r+p-1}, \Gamma_{r+p}\right) \\
& \left.\times d \mathbf{P}\left(S_{p}^{(1)} \leqslant x_{r+p}, p=1, \ldots, k-r\right)\right) \\
= & \int_{D_{1}} \ldots \int_{D_{r}} \prod_{i=1}^{r} G\left(x_{i}-x_{i-1}, \Gamma_{i}\right) \\
& \times\left(\int_{D_{r+1}-x_{r}} \ldots \int_{D_{k}-x_{r}} \prod_{p=1}^{k-r} G\left(x_{r+p}-x_{r+p-1}, \Gamma_{r+p}\right)\right. \\
& \left.\times d \mathbf{P}\left(S_{p}^{(1)} \leqslant x_{r+p}, p=1, \ldots, k-r\right)\right) d \mathbf{P}\left(S_{p} \leqslant x_{i}, i=1, \ldots, r, \tau_{j}=r\right) \\
= & \int_{D_{1}} \ldots \int_{D_{k}} \prod_{i=1}^{k} G\left(x_{i}-x_{i-1}, \Gamma_{i}\right) \\
& \quad \times d \mathbf{P}\left(S_{i} \leqslant x_{i}, i=1, \ldots, r, ; \tau_{j}=r, S_{r}+S_{p}^{(1)} \leqslant x_{r+p}, p=1, \ldots, k-r\right)
\end{aligned}
$$


(здесь $\chi_{A}$ - индикатор множества $A$, при нахождении интеграла по множествам $D_{r+1}-S_{r}, \ldots, D_{k}-S_{r}$ случайная величина $S_{r}$ фиксируется). Складывая (16) при разных $r$ и (15) и учитывая, что ввиду (14)

$$
\begin{aligned}
\mathbf{P}\left(S_{i}^{(j)} \in D_{i}, i=1, \ldots, k\right) & \\
=\sum_{r=1}^{k} \mathbf{P}\left(S_{i} \in D_{i}, i=1, \ldots, r, \tau_{j}=r, S_{r}+\right. & \left.S_{p}^{(1)} \in D_{r+p}, p=1, \ldots, k-r\right) \\
& +\mathbf{P}\left(S_{i} \in D_{i}, i=1, \ldots, k, k<\tau_{j}\right),
\end{aligned}
$$

получаем соотношение (8). Лемма 1 доказана.

Введем еще две сопровождающие случайные последовательности, порожденные случайной средой:

$$
\begin{aligned}
a_{n} & =e^{-S_{n}}, & & n \in \mathbf{N}_{0}, \\
b_{0}=0, & b_{n}=\sum_{i=0}^{n-1} \eta_{i+1} e^{-S_{i}}, & & n \in \mathbf{N} .
\end{aligned}
$$

В работе автора [5] установлено, что если $M X_{1}=0$ и выполнено условие (a), то при любом фиксированном $j \in \mathbf{N}_{0}$ и $n \rightarrow \infty$

$$
\begin{aligned}
& \left\{a_{[n t]}, t \in(0,1] \mid \tau_{j} \leqslant n<\tau_{j+1}\right\} \Rightarrow 0, \\
& \left\{b_{[n t]}, t \in(0,1] \mid \tau_{j} \leqslant n<\tau_{j+1}\right\} \Rightarrow \nu_{j},
\end{aligned}
$$

где $\nu_{j}$ - случайный процесс с положительными постоянными реализациями на $(0,1]$, знак $\Rightarrow$ означает сходимость процессов в смысле конечномерных распределений.

Лемма 2. Если $\mathrm{M} X_{1}=0$ и выполнены условия (a), (b), то при любых фиксированных $j \in \mathbf{N}_{0} u \lambda \in(0,1)$

$$
\begin{array}{r}
\lim _{m \rightarrow \infty} \lim _{n \rightarrow \infty} \frac{\mathbf{P}\left(\tau_{j} \leqslant \lambda m, n<\tau_{j+1}\right)}{\mathbf{P}\left(\tau_{j} \leqslant n<\tau_{j+1}\right)}=1, \\
\lim _{m \rightarrow \infty} \varlimsup_{n \rightarrow \infty} \mathbf{M}\left(b_{n}-b_{m} \mid \tau_{j} \leqslant \lambda m, n<\tau_{j+1}\right)=0, \\
\varlimsup_{m \rightarrow \infty} \varlimsup_{n \rightarrow \infty} \mathbf{M}\left(a_{n}-a_{m} \mid \tau_{j} \leqslant \lambda m, n<\tau_{j+1}\right)=0 .
\end{array}
$$

Замечание 1. Соотношение (20) обобщает указанное в [4] и уточненное в работе [6] утверждение: если $M X_{1}=0$ и выполнено условие (a), то

$$
\lim _{m \rightarrow \infty} \varlimsup_{n \rightarrow \infty} \mathbf{M}\left(b_{n}-b_{m} \mid \tau_{1}>n\right)=0 .
$$

Доказательство леммы 2. Зафиксируем $j \in \mathbf{N}$ и положим

$$
\begin{aligned}
& S_{0}^{\prime}=0, \quad S_{i}^{\prime}=S_{\tau_{j}+i}-S_{\tau_{j}}, \quad \eta_{i}^{\prime}=\eta_{\tau_{j}+i}, \quad i \in \mathbf{N}, \\
& \tau_{1}^{\prime}=\tau_{j+1}-\tau_{j}, \\
& b_{0}^{\prime}=0, \quad b_{n}^{\prime}=\sum_{i=0}^{n-1} \eta_{i+1}^{\prime} e^{-S_{i}^{\prime}}, \quad n \in \mathbf{N} \text {. }
\end{aligned}
$$


Будем считать, что $n>m$. Поскольку

$$
\begin{aligned}
\mathbf{P}\left(\tau_{j} \leqslant \lambda m, n<\tau_{j+1}\right) & =\sum_{k=j}^{[\lambda m]} \mathbf{P}\left(\tau_{j}=k, \tau_{1}^{\prime}>n-k\right) \\
& =\sum_{k=j}^{[\lambda m]} \mathbf{P}\left(\tau_{j}=k\right) \mathbf{P}\left(\tau_{1}^{\prime}>n-k\right),
\end{aligned}
$$

в силу соотношения (3)

$$
\lim _{n \rightarrow \infty} \sqrt{n} \mathbf{P}\left(\tau_{j} \leqslant \lambda m, n<\tau_{j+1}\right)=c_{1} \mathbf{P}\left(\tau_{j} \leqslant \lambda m\right) .
$$

Но $\tau_{j}<\infty$ п. н., поэтому, снова учитывая (3), получаем соотношение (19).

Установим теперь соотношение (20). При $\tau_{j} \leqslant \lambda m$ справедливо представление

$$
b_{n}-b_{m}=e^{-S_{\tau_{j}}}\left(b_{n-\tau_{j}}^{\prime}-b_{m-\tau_{j}}^{\prime}\right) \text {. }
$$

Следовательно,

$$
\begin{aligned}
& \mathbf{M}\left(b_{n}-b_{m} \mid \tau_{j} \leqslant \lambda m, n<\tau_{j+1}\right) \\
& \quad=\mathbf{P}\left(\tau_{j} \leqslant \lambda m, n<\tau_{j+1}\right)^{-1} \sum_{k=j}^{[\lambda m]} \mathbf{M}\left[e^{-S_{\tau_{j}}}\left(b_{n-k}^{\prime}-b_{m-k}^{\prime}\right) ; \tau_{j}=k, \tau_{1}^{\prime}>n-k\right] .
\end{aligned}
$$

Случайные величины со штрихами распределены так же, как соответствующие случайные величины без штрихов, и не зависят от $\tau_{j}, S_{\tau_{j}}$, поэтому

$$
\begin{aligned}
\mathrm{M}\left[e^{-S_{\tau_{j}}}\left(b_{n-k}^{\prime}-b_{m-k}^{\prime}\right) ; \tau_{j}=k, \tau_{1}^{\prime}\right. & >n-k] \\
& =\mathrm{M}\left(e^{-S_{\tau_{j}}} \tau_{j}=k\right) \mathrm{M}\left(b_{n-k}-b_{m-k} ; \tau_{1}>n-k\right) .
\end{aligned}
$$

Из соотношений (22), (23) следует, что

$$
\begin{aligned}
& \mathbf{M}\left(b_{n}-b_{m} \mid \tau_{j} \leqslant \lambda m, n<\tau_{j+1}\right) \\
& \quad=\mathbf{P}\left(\tau_{j} \leqslant \lambda m, n<\tau_{j+1}\right)^{-1} \sum_{k=j}^{[\lambda m]} \mathbf{M}\left(e^{-S_{\tau_{j}}} \tau_{j}=k\right) \mathbf{M}\left(b_{n-k}-b_{m-k} ; \tau_{1}>n-k\right) .
\end{aligned}
$$

Ввиду замечания 1 для произвольного $\varepsilon \in(0, \infty)$ существует такое $M \in \mathbf{N}$, что при всех $m>M$ справедливо неравенство

$$
\varlimsup_{n \rightarrow \infty} \mathbf{M}\left(b_{n}-b_{m} \mid \tau_{1}>n\right) \leqslant \varepsilon .
$$

Из соотношений $(24),(25)$ вытекает, что при всех $m>M /(1-\lambda)$

$$
\varlimsup_{n \rightarrow \infty} \mathbf{M}\left(b_{n}-b_{m} \mid \tau_{j} \leqslant \lambda m, n<\tau_{j+1}\right) \leqslant \varepsilon M e^{-S_{\tau_{j}}} \varlimsup_{n \rightarrow \infty} \frac{\mathbf{P}\left(\tau_{1}>n-\lambda m\right)}{\mathbf{P}\left(\tau_{j} \leqslant \lambda m, n<\tau_{j+1}\right)} .
$$

Отсюда, применяя соотношения (3) и (19), находим, что

$$
\varlimsup_{m \rightarrow \infty} \varlimsup_{n \rightarrow \infty} \mathbf{M}\left(b_{n}-b_{m} \mid \tau_{j} \leqslant \lambda m, n<\tau_{j+1}\right) \leqslant \varepsilon M e^{-S_{\tau_{j}}} .
$$

Вследствие условия (b) последнее математическое ожидание конечно (см. [1]), поэтому ввиду произвольности $\varepsilon$ приходим к соотношению (20). Соотношение (21) является следствием (20) (надо положить $\eta_{1}=\eta_{2}=\ldots=1$ ). Лемма 2 доказана. 
Аналогично устанавливается следующее утверждение (см. [1]).

Лемма 3. Если $\mathrm{M} X_{1}=0$ и выполнены условия (a), (b), то при любых фиксированных $j \in \mathbf{N}, \lambda u t$ таких, что $0<\lambda<t<1$, справедливы соотношения

$$
\begin{gathered}
\lim _{n \rightarrow \infty} M\left(b_{n}-b_{[n t]} \mid \tau_{j} \leqslant \lambda n, n<\tau_{j+1}\right)=0, \\
\lim _{n \rightarrow \infty} M\left(a_{n}-a_{[n t]} \mid \tau_{j} \leqslant \lambda n, n<\tau_{j+1}\right)=0 .
\end{gathered}
$$

3. Перейдем к непосредственному рассмотрению ветвящегося процесса в случайной среде.

Лемма 4. Если $\left\{\xi_{n}\right\}$ - критический ветвящийся прочесс в случайной среде $\left\{\pi_{n}\right\}$ $u 0<\mathbf{M} X_{1}^{2}<\infty$, то при любом фиксированном $j \in \mathbf{N}_{0} u n \rightarrow \infty$

$$
\left\{\pi_{i}, i \in \mathbf{N}_{0} \mid \tau_{j} \leqslant n<\tau_{j+1}\right\} \stackrel{\mathscr{D}}{\longrightarrow}\left\{\pi_{i}^{(j)}, \in \mathbf{N}_{0}\right\}
$$

где $\left\{\pi_{i}^{(j)}, i \in \mathbf{N}_{0}\right\}$ - некоторал случайная среда.

Замечание 2. Пусть

$$
\pi_{i}^{(j)}=\left\{\pi_{i}^{(j)}(0), \pi_{i}^{(j)}(1), \ldots\right\}
$$

и при $k \in \mathbf{N}_{0}$

$$
\begin{aligned}
\pi_{i, k} & =\left(\pi_{i}(0), \pi_{i}(1), \ldots, \pi_{i}(k)\right) \\
\pi_{i, k}^{(j)} & =\left(\pi_{i}^{(j)}(0), \pi_{i}^{(j)}(1), \ldots, \pi_{i}^{(j)}(k)\right)
\end{aligned}
$$

Соотношение (26) надо понимать следующим образом: при любом фиксированном $k \in \mathbf{N}_{0}$ и $n \rightarrow \infty$

$$
\left\{\pi_{i, k}, i \in \mathbf{N}_{0} \mid \tau_{j} \leqslant n<\tau_{j+1}\right\} \stackrel{D}{\longrightarrow}\left\{\pi_{i, k}^{(j)}, \in \mathbf{N}\right\}
$$

Доказательство леммъ 4. Рассмотрим при фиксированном $k \in \mathbf{N}_{0}$ последовательность случайных векторов

$$
\left(X_{1} ; \pi_{0, k}\right),\left(X_{2} ; \pi_{1, k}\right), \ldots
$$

Ясно, что эти случайные векторы независимы и одинаково распределены. В силу леммы 1 при любом фиксированном $j \in \mathbf{N}_{0}$ и $n \rightarrow \infty$

$$
\left\{S_{1} ; \pi_{0, k} ; S_{2} ; \pi_{1, k} ; \ldots \mid \tau_{j} \leqslant n<\tau_{j+1}\right\} \stackrel{\mathscr{D}}{\longrightarrow}\left\{S_{1}^{(j)} ; \pi_{0, k}^{(j)} ; S_{2}^{(j)} ; \pi_{2, k}^{(j)} ; \ldots\right\},
$$

где $\pi_{0, k}^{(j)}, \pi_{2, k}^{(j)}, \ldots$ - некоторые случайные векторы. Ясно, что при любых $i, j, k \in \mathbf{N}_{0}$ первые $k+1$ компонент вектора $\pi_{i, k+1}^{(j)}$ можно считать совпадающими п. н. с соответствующими компонентами вектора $\pi_{i, k}^{(j)}$. Поэтому последовательность случайных векторов $\left\{\pi_{i, k}^{(j)}, k \in \mathbf{N}_{0}\right\}$ порождает случайную последовательность

$$
\pi_{i}^{(j)}=\left\{\pi_{i}^{(j)}(0), \pi_{i}^{(j)}(1), \ldots\right\}
$$


и имеет место соотношение (26). Покажем, что последовательность $\left\{\pi_{i}^{(j)}, i \in \mathbf{N}_{0}\right\}$ образует случайную среду. Очевидно, $\pi_{i}^{(j)}(l) \geqslant 0$ при любом $l \in \mathbf{N}_{0}$. Установим, что при любых $i, j \in \mathbf{N}_{0}$

$$
\sum_{l=0}^{\infty} \pi_{i}^{(j)}(l)=1
$$

В силу леммы 1 при $L \in \mathbf{N}_{0}$ и $\varepsilon \in(0,1)$

$$
\begin{gathered}
\mathbf{P}\left(\sum_{l=0}^{L} \pi_{i}^{(j)}(l) \geqslant 1-\varepsilon\right)=\int_{-\infty}^{\infty} \ldots \int_{-\infty}^{\infty} \mathbf{P}\left(\sum_{l=0}^{L} \pi_{i}(l) \geqslant 1-\varepsilon \mid X_{i+1}=x_{i+1}-x_{i}\right) \\
\times d \mathbf{P}\left(S_{r}^{(j)} \leqslant x_{r}, r=1, \ldots, i+1\right) .
\end{gathered}
$$

Переходя к пределу при $L \rightarrow \infty$, по теореме о монотонной сходимости получаем, что

$$
\begin{aligned}
\mathbf{P}\left(\sum_{l=0}^{\infty} \pi_{i}^{(j)}(l) \geqslant 1-\varepsilon\right)= & \int_{-\infty}^{\infty} \ldots \int_{-\infty}^{\infty} \mathbf{P}\left(\sum_{l=0}^{\infty} \pi_{i}(l) \geqslant 1-\varepsilon \mid X_{i+1}=x_{i+1}-x_{i}\right) \\
& \times d \mathbf{P}\left(S_{r}^{(j)} \leqslant x_{r}, r=1, \ldots, i+1\right) \\
= & \int_{-\infty}^{\infty} \ldots \int_{-\infty}^{\infty} d \mathbf{P}\left(S_{r}^{(j)} \leqslant x_{r}, r=1, \ldots, i+1\right)=1 .
\end{aligned}
$$

С другой стороны, с вероятностью 1

$$
\sum_{l=0}^{L} \pi_{i}(l) \leqslant 1 \Rightarrow \sum_{l=0}^{L} \pi_{i}^{(j)}(l) \leqslant 1 \Rightarrow \sum_{l=0}^{\infty} \pi_{i}^{(j)}(l) \leqslant 1 .
$$

Из соотношений (29), (30) следует (28). Лемма 4 доказана.

Компоненты случайной среды $\left\{\pi_{i}^{(j)}\right\}$ не являются, вообще говоря, независимыми одинаково распределенными случайными последовательностями. Тем не менее, зададим ветвящийся процесс $\left\{\xi_{i}^{(j)}, i \in \mathbf{N}_{0}\right\}$ в случайной среде $\left\{\pi_{i}^{(j)}, i \in \mathbf{N}_{0}\right\}$ в соответствии с соотношением (1).

Лемма 5. Если $\left\{\xi_{n}\right\}$ - критический ветвящийся проџесс в случайной среде и $0<\mathrm{M} X_{1}^{2}<\infty$, то при любом фиксированном $j \in \mathbf{N}_{0}$ u $n \rightarrow \infty$

$$
\left\{\xi_{i}, i \in \mathbf{N}_{0} \mid \tau_{j} \leqslant n<\tau_{j+1}\right\} \stackrel{\mathscr{D}}{\longrightarrow}\left\{\xi_{i}^{(j)}, i \in \mathbf{N}_{0}\right\}
$$

Доказательство. Зафиксируем $k \in \mathbf{N}$ и $l_{1}, \ldots, l_{k} \in \mathbf{N}_{0}$. Положим

$$
l=\max \left(l_{1}, \ldots, l_{k}\right)
$$

Очевидно,

$$
\mathbf{P}_{\pi}\left(\xi_{1}=l_{1}, \ldots, \xi_{k}=l_{k}\right)=f\left(\pi_{0, l} ; \pi_{1, l} ; \ldots ; \pi_{k-1, l}\right)
$$


где $f-$ некоторая неслучайная непрерывная и ограниченная функция на $[0,1]^{(l+1) k}$. Поэтому

$$
\begin{aligned}
\mathbf{P}\left(\xi_{1}\right. & \left.=l_{1}, \ldots, \xi_{k}=l_{k} \mid \tau_{j} \leqslant n<\tau_{j+1}\right) \\
& =\frac{1}{\mathbf{P}\left(\tau_{j} \leqslant n<\tau_{j+1}\right)} \mathbf{M}\left(\chi_{\left\{\xi_{1}=l_{1}, \ldots, \xi_{k}=l_{k}\right\}} \chi_{\left\{\tau_{j} \leqslant n<\tau_{j+1}\right\}}\right) \\
& =\frac{1}{\mathbf{P}\left(\tau_{j} \leqslant n<\tau_{j+1}\right)} \mathbf{M}\left(\chi_{\left\{\tau_{j} \leqslant n<\tau_{j+1}\right\}} \mathbf{P}_{\pi}\left(\xi_{1}=l_{1}, \ldots, \xi_{k}=l_{k}\right)\right) \\
& =\mathbf{M}\left(f\left(\pi_{0, l} ; \pi_{1, l} ; \ldots ; \pi_{k-1, l}\right) \mid \tau_{j} \leqslant n<\tau_{j+1}\right) .
\end{aligned}
$$

Отсода, учитывая лемму 1 , получаем, что

$$
\lim _{n \rightarrow \infty} \mathbf{P}\left(\xi_{1}=l_{1}, \ldots, x_{k}=l_{k} \mid \tau_{j} \leqslant n<\tau_{j+1}\right)=\mathbf{M} f\left(\pi_{0, l}^{(j)} ; \pi_{1, l}^{(j)} ; \ldots ; \pi_{k-1, l}^{(j)}\right) .
$$

Ясно, что $f\left(\pi_{0, l}^{(j)} ; \pi_{1, l}^{(j)} ; \ldots ; \pi_{k-1, l}^{(j)}\right)$ имеет тот же смысл для случайной среды $\left\{\pi_{i}^{(j)}, i \in \mathbf{N}_{0}\right\}$, что и $f\left(\pi_{0, l} ; \pi_{1, l} ; \ldots ; \pi_{k-1, l}\right)$ для среды $\left\{\pi_{i}, i \in \mathbf{N}_{0}\right\}$, то есть

$$
f\left(\pi_{0, l}^{(j)} ; \pi_{1, l}^{(j)} ; \ldots ; \pi_{k-1, l}^{(j)}\right)=\mathbf{P}_{\pi^{(j)}}\left(\xi_{1}^{(j)}=l_{1}, \ldots, \xi_{k}^{(j)}=l_{k}\right),
$$

где $\mathbf{P}_{\pi^{(j)}}$ означает вероятность, вычисленную при условии, что среда $\left\{\pi_{i}^{(j)}, i \in \mathbf{N}_{0}\right\}$ фиксирована. Из соотношений (31), (32) следует, что

$$
\lim _{n \rightarrow \infty} \mathbf{P}\left(\xi_{1}=l_{1}, \ldots, \xi_{k}=l_{k} \mid \tau_{j} \leqslant n<\tau_{j+1}\right)=\mathbf{P}\left(\xi_{1}^{(j)}=l_{1}, \ldots, \xi_{k}^{(j)}=l_{k}\right) .
$$

Лемма 5 доказана.

Наряду со случайной средой $\left\{\pi_{i}^{(j)}, i \in \mathbf{N}_{0}\right\}$ у нас имеется случайная последовательность $\left\{S_{i}^{(j)}, i \in \mathbf{N}_{0}\right\}$ (см. (7)). Оказывается, что при любом $j \in \mathbf{N}_{0}$ последовательность $\left\{S_{i}^{(j)}, i \in \mathbf{N}_{0}\right\}$ п. н. совпадает с сопровождающим случайным блужданием процесса $\left\{\xi_{i}^{(j)}, i \in \mathbf{N}_{0}\right\}$. Положим

$$
\begin{aligned}
f_{n, j}(s) & =\sum_{i=0}^{\infty} \pi_{n}^{(j)}(i) s^{i}, s \in[-1,1], \quad n \in \mathbf{N}_{0} \\
X_{n}^{(j)} & =\ln f_{n-1, j}^{\prime}(1), \quad . \quad n \in \mathbf{N} .
\end{aligned}
$$

Утверждается, что для любого $i \in \mathbf{N}$ п. н.

$$
S_{i}^{(j)}=\sum_{n=1}^{i} X_{n}^{(j)}
$$

Ради краткости изложения покажем, например, что $S_{1}^{(j)}=X_{1}^{(j)}$ п. н., что равносильно соотношению

$$
\exp S_{1}^{(j)}=\exp X_{1}^{(j)} \quad \text { п. н. }
$$

Очевидно, что для каждого $m \in \mathbf{N}$

$$
\sum_{i=1}^{m} i \pi_{0}(i) \leqslant \exp S_{1} \quad \text { п.н. } \Rightarrow \sum_{i=1}^{m} i \pi_{0}^{(j)}(i) \leqslant \exp S_{1}^{(j)} \text { п.н. }
$$


Поэтому

$$
\exp X_{1}^{(j)}=\sum_{i=1}^{\infty} i \pi_{0}^{(j)}(i) \leqslant \exp S_{1}^{(j)} \quad \text { п.н.. }
$$

Если мы покажем, что для каждого $L \in(0, \infty)$

$$
\mathbf{M}\left(\left(\exp X_{1}^{(j)}\right) \wedge L\right)=\mathbf{M}\left(\left(\exp S_{1}^{(j)}\right) \wedge L\right),
$$

то отсюда будет следовать (34). В силу леммы 1 при каждом $m \in \mathbf{N}$

$$
\begin{aligned}
\mathbf{M}\left(\left(\sum_{i=0}^{m} i \pi_{0}^{(j)}(i)\right) \wedge L\right) & =\int_{-\infty}^{\infty} \mathbf{M}\left(\left(\sum_{i=0}^{m} i \pi_{0}^{(j)}(i)\right) \wedge L \mid S_{1}^{(j)}=x\right) d \mathbf{P}\left(S_{1}^{(j)} \leqslant x\right) \\
& =\int_{-\infty}^{\infty} \mathbf{M}\left(\left(\sum_{i=0}^{m} i \pi_{0}(i)\right) \wedge L \mid S_{1}=x\right) d \mathbf{P}\left(S_{1}^{(j)} \leqslant x\right) .
\end{aligned}
$$

Переходя к пределу при $m \rightarrow \infty$, по теореме о монотонной сходимости получаем, что

$$
\begin{aligned}
\mathbf{M}\left(\left(\exp X_{1}^{(j)}\right) \wedge L\right) & =\mathbf{M}\left(\left(\sum_{i=0}^{\infty} i \pi_{0}^{(j)}(i)\right) \wedge L\right) \\
& =\int_{-\infty}^{\infty} \mathbf{M}\left(\left(\sum_{i=0}^{\infty} i \pi_{0}(i)\right) \wedge L \mid S_{1}=x\right) d \mathbf{P}\left(S_{1}^{(j)} \leqslant x\right) \\
& =\int_{-\infty}^{\infty} \mathbf{M}\left(\left(\exp S_{1}\right) \wedge L \mid S_{1}=x\right) d \mathbf{P}\left(S_{1}^{(j)} \leqslant x\right) \\
& =\mathbf{M}\left(\left(\exp S_{1}^{(j)}\right) \wedge L\right) .
\end{aligned}
$$

Итак, соотношение (35), а следовательно, и (34) доказаны.

Вместо соотношения (27) можно написать, что при $n \rightarrow \infty$

$$
\left\{S_{1} ; \pi_{0, k} ; \eta_{1} ; S_{2} ; \pi_{1, k} ; \eta_{2} ; \ldots \mid \tau_{j} \leqslant n<\tau_{j+1}\right\} \stackrel{\mathscr{D}}{\longrightarrow}\left\{S_{1}^{(j)} ; \pi_{0, k}^{(j)} ; \eta_{1}^{(j)} ; S_{2}^{(j)} ; \pi_{1, k}^{(j)} ; \eta_{2}^{(j)} ; \ldots\right\} .
$$

Аналогично (33) можно показать, что п. н.

$$
\eta_{n}^{(j)}=\frac{f_{n-1, j}^{\prime \prime}(1)}{2\left(f_{n-1, j}^{\prime}(1)\right)^{2}}, \quad n \in \mathbf{N} .
$$

Отсюда следует, что сопровождающие случайные последовательности $\left\{S_{i}^{(j)}\right\},\left\{a_{i}^{(j)}\right\}$, $\left\{b_{i}^{(j)}\right\}$ для случайного процесса $\left\{\xi_{i}^{(j)}\right\}$ являются пределами по распределению при $n \rightarrow \infty$ соответствующих сопровождающих случайных последовательностей $\left\{S_{i}\right\}$, $\left\{a_{i}\right\},\left\{b_{i}\right\}$ процесса $\left\{\xi_{i}\right\}$, рассматриваемых при условии $\left\{\tau_{j} \leqslant n<\tau_{j+1}\right\}$.

С учетом сказанного и соотношения $m_{i}=\exp S_{i}, i \in \mathbf{N}_{0}$, аналогично лемме 5 устанавливается следующее утверждение.

Лемма 6. Если $\left\{\xi_{n}\right\}$ - критический ветвящийся прочесс в случайной среде и $0<\mathrm{M} X_{1}^{2}<\infty$, то при любом фиксированном $j \in \mathrm{N}_{0} u n \rightarrow \infty$

$$
\left\{\xi_{i} / m_{i}, i \in \mathbf{N}_{0} \mid \tau_{j} \leqslant n<\tau_{j+1}\right\} \stackrel{\mathscr{D}}{\longrightarrow}\left\{\xi_{i}^{(j)} / m_{i}^{(j)}, i \in \mathbf{N}_{0}\right\}
$$

əде $m_{i}^{(j)}=\mathbf{M}_{\pi^{(j)}} \xi_{i}^{(j)}$. 
Следующее утверждение, доказанное в работе [1], частично объясняет важную роль, которую играют сопровождающие случайные последовательности $\left\{a_{n}\right\}$ и $\left\{b_{n}\right\}$ при изучении ветвящихся процессов в случайной среде.

Лемма 7. Пусть $\left\{\xi_{n}\right\}$ - ветвящийсл прочесс в случайной среде $\left\{\pi_{n}\right\}$ u пусть $c$ вероятностъю 1 случайные величины $X_{1}, \eta_{1}$ конечны, тогда при любых $k, l \in \mathbf{N}_{0}$, $l \geqslant k$, справедливо соотношение

$$
\mathbf{M}_{\pi}\left(\frac{\xi_{l}}{m_{l}}-\frac{\xi_{k}}{m_{k}}\right)^{2}=2\left(b_{l}-b_{k}\right)+a_{l}-a_{k} .
$$

Лемма 8. Если $\left\{\xi_{n}\right\}-$ - критический ветвящийся прочесс в случайной среде и въполнено условие (a), то при любом фиксированном $j \in \mathbf{N}_{0} u i \rightarrow \infty$

$$
\frac{\xi_{i}^{(j)}}{m_{i}^{(j)}} \stackrel{\mathscr{D}}{\longrightarrow} \mu^{(j)}
$$

где $\mu^{(j)}$ - некоторая неотрицательная случайная величина, для которой $\mathbf{P}\left(\mu^{(0)}>0\right)>0$.

Доказателъство. При фиксированной среде $\left\{\pi_{i}^{(j)}, i \in \mathbf{N}_{0}\right\}$ случайная последовательность $\left\{\xi_{i}^{(j)} / m_{i}^{(j)}, i \in \mathbf{N}_{0}\right\}$ является мартингалом и поэтому п. н. существует предел этой последовательности, который мы будем обозначать $\mu_{\mathbf{P}_{\pi}^{(j)}}$. Это означает, что при фиксированной среде $\left\{\pi_{i}^{(j)}, i \in \mathbf{N}_{0}\right\}$ последовательность $\left\{\xi_{i}^{(j)} / m_{i}^{(j)}, i \in \mathbf{N}_{0}\right\}$ фундаментальна с вероятностью 1. Отсюда следует (уже без фиксирования среды), что последовательность $\left\{\xi_{i}^{(j)} / m_{i}^{(j)}, i \in \mathbf{N}_{0}\right\}$ фундаментальна и, следовательно, п. н. существует

$$
\lim _{i \rightarrow \infty} \frac{\xi_{i}^{(j)}}{m_{i}^{(j)}}=\mu^{(j)}
$$

где $\mu^{(j)}$ - некоторая неотрицательная случайная величина. Покажем, что вероятность $\mathbf{P}\left(\mu^{(0)}>0\right)$ положительна. В [4] в условиях доказываемой леммы установлено, что с вероятностью 1

$$
\sum_{i=0}^{\infty} \eta_{i+1}^{(0)} e^{-S_{i}^{(0)}}<\infty, \quad \lim _{i \rightarrow \infty} S_{i}^{(0)}=\infty .
$$

Значит, п.н.

$$
\sum_{i=0}^{\infty}\left(2 \eta_{i+1}^{(0)} e^{-S_{i}^{(0)}}+e^{-S_{i+1}^{(0)}}-e^{-S_{i}^{(0)}}\right)<\infty .
$$

Отсюда ввиду леммы 7 следует, что

$$
\begin{aligned}
\lim _{k, l \rightarrow \infty, l>k} \mathbf{M}_{\pi^{(0)}}\left(\frac{\xi_{l}^{(0)}}{m_{l}^{(0)}}-\frac{\xi_{k}^{(0)}}{m_{k}^{(0)}}\right)^{2} & =\lim _{k, l \rightarrow \infty, l>k}\left(2\left(b_{l}^{(0)}-b_{k}^{(0)}\right)+a_{l}^{(0)}-a_{k}^{(0)}\right) \\
& =\lim _{k, l \rightarrow \infty, l>k} \sum_{i=k}^{l-1}\left(2 \eta_{i+1}^{(0)} e^{-S_{i}^{(0)}}+e^{-S_{i+1}^{(0)}}-e^{-S_{i}^{(0)}}\right)=0 .
\end{aligned}
$$


Итак, последовательность $\left\{\xi_{i}^{(0)} / m_{i}^{(0)}, i \in \mathbf{N}_{0}\right\}$ при фиксированной среде $\left\{\pi_{i}^{(0)}, i \in \mathbf{N}_{0}\right\}$ сходится в среднем квадратическом. Поэтому

$$
\lim _{i \rightarrow \infty} \mathbf{M}_{\pi^{(0)}} \frac{\xi_{i}^{(0)}}{m_{i}^{(0)}}=\mathbf{M}_{\pi^{(0)}} \mu_{\pi^{(0)}}
$$

и, следовательно, $\mathbf{M}_{\pi^{(0)}} \mu_{\pi^{(0)}}=1$. Но это означает, что $\mathbf{P}_{\pi^{(0)}}\left(\mu_{\pi^{0}}>0\right)>0$. Отсюда вытекает, что

$$
\mathbf{P}_{\pi^{(0)}}\left(\bigcup_{k \in \mathbf{N}} \bigcup_{n \in \mathbf{N}} \bigcap_{i \geqslant n}\left\{\frac{\xi_{i}^{(0)}}{m_{i}^{(0)}}>\frac{1}{k}\right\}\right)>0
$$

и, следовательно,

$$
\mathbf{P}\left(\bigcup_{k \in \mathbf{N}} \bigcup_{n \in \mathbf{N}} \bigcap_{i \geqslant n}\left\{\frac{\xi_{i}^{(0)}}{m_{i}^{(0)}}>\frac{1}{k}\right\}\right)>0 .
$$

Из последнего неравенства получаем, что $\mathbf{P}\left(\mu^{(j)}>0\right)>0$. Лемма 8 доказана.

Лемма 9. В условиях теоремы 1 при любом фиксированном $j \in \mathbf{N}_{0} u n \rightarrow \infty$

$$
\left\{\xi_{n} / m_{n} \mid \tau_{j} \leqslant n<\tau_{j+1}\right\} \stackrel{\mathscr{D}}{\longrightarrow} \mu^{(j)},
$$

где $\mu^{(j)}$ - та же случайная величина, что и в лемме 8.

Доказательство. Покажем, что для произвольного $\varepsilon \in(0, \infty)$

$$
\lim _{i \rightarrow \infty} \varlimsup_{n \rightarrow \infty} \mathbf{P}\left(\left|\frac{\xi_{n}}{m_{n}}-\frac{\xi_{i}}{m_{i}}\right| \geqslant \varepsilon \mid \tau_{j} \leqslant n<\tau_{j+1}\right)=0 .
$$

Действительно, при $\lambda \in(0,1)$

$$
\begin{aligned}
\mathbf{P}\left(\left|\frac{\xi_{n}}{m_{n}}-\frac{\xi_{i}}{m_{i}}\right| \geqslant \varepsilon ; \tau_{j} \leqslant n<\tau_{j+1}\right) & \\
& \leqslant \mathbf{P}\left(\lambda i<\tau_{j}<n<\tau_{j+1}\right)+\mathbf{P}\left(\left|\frac{\xi_{n}}{m_{n}}-\frac{\xi_{i}}{m_{i}}\right| \geqslant \varepsilon ; \tau_{j} \leqslant \lambda i, n<\tau_{j+1}\right) .
\end{aligned}
$$

Рассмотрим второе слагаемое в правой части (37). По неравенству Чебышева

$$
\begin{aligned}
\mathbf{P}\left(\left|\frac{\xi_{n}}{m_{n}}-\frac{\xi_{i}}{m_{i}}\right| \geqslant \varepsilon ;\right. & \left.\tau_{j} \leqslant \lambda i, n<\tau_{j+1}\right) \\
= & \mathbf{M}\left(\mathbf{P}_{\pi}\left(\left|\frac{\xi_{n}}{m_{n}}-\frac{\xi_{i}}{m_{i}}\right| \geqslant \varepsilon\right) ; \tau_{j} \leqslant \lambda i, n<\tau_{j+1}\right) \\
& \leqslant \frac{1}{\varepsilon^{2}} \mathbf{M}\left(\mathbf{M}_{\pi}\left(\frac{\xi_{n}}{m_{n}}-\frac{\xi_{i}}{m_{i}}\right)^{2} ; \tau_{j} \leqslant \lambda i, n<\tau_{j+1}\right) .
\end{aligned}
$$

Отсюда, применяя лемму 7 , находим, что

$$
\begin{aligned}
\mathbf{P}\left(\left|\frac{\xi_{n}}{m_{n}}-\frac{\xi_{i}}{m_{i}}\right| \geqslant \varepsilon ; \tau_{j} \leqslant \lambda i, n\right. & \left.<\tau_{j+1}\right) \\
& \leqslant \frac{1}{\varepsilon^{2}} \mathbf{M}\left(2\left(b_{n}-b_{i}\right)+a_{n}-a_{i} ; \tau_{j} \leqslant \lambda i, n<\tau_{j+1}\right) .
\end{aligned}
$$


Из соотношений (37) и (38) вытекает, что при $\lambda \in(0,1)$

$$
\begin{aligned}
\mathbf{P}\left(\left|\frac{\xi_{n}}{m_{n}}-\frac{\xi_{i}}{m_{i}}\right| \geqslant \varepsilon ; \tau_{j} \leqslant n<\tau_{j+1}\right) & \\
& \leqslant \mathbf{P}\left(\lambda i<\tau_{j}<n<\tau_{j+1}\right)+\frac{1}{\varepsilon^{2}} \mathbf{M}\left(2\left(b_{n}-b_{i}\right)+a_{n}-a_{i} ; \tau_{j} \leqslant \lambda i, n<\tau_{j+1}\right) .
\end{aligned}
$$

Применяя теперь лемму 2 , получаем соотношение (36). Из лемм 6,8 и соотношения (36) следует (см. параграф 4 в [7]) утверждение леммы 9.

Г. Керстинг и И. Гейгер [8] установили, что если $\left\{\xi_{n}\right\}-$ критический ветвящийся процесс и выполнено условие (а), то при $n \rightarrow \infty$

$$
\mathbf{P}(T>n) \sim \frac{c_{2}}{\sqrt{n}}
$$

где $c_{2}$ - положительная постоянная.

Лемма 10. Пусть $\left\{\xi_{n}\right\}$ - критический ветвящийся прочесс в случайной среде и выполнено условие (а). Если $\left\{A_{n}\right\}$ - такая последователъность случайных событий, что при любом фиксированном $j \in \mathbf{N}_{0}$ существует

$$
\lim _{n \rightarrow \infty} \mathbf{P}\left(A_{n}, T>n \mid \tau_{j} \leqslant n<\tau_{j+1}\right)=a_{j}
$$

то существует

$$
\lim _{n \rightarrow \infty} \mathbf{P}\left(A_{n} \mid T>n\right)=\frac{c_{1}}{c_{2}} \sum_{j=0}^{\infty} a_{j}
$$

әде $c_{1}, c_{2}$ - положительные постояннъе из соотношений (3) и (39) соответственно.

Доказателъство. Справедливо представление

$$
\mathbf{P}\left(A_{n} \mid T>n\right)=\sum_{j=0}^{\infty} \frac{\mathbf{P}\left(\tau_{j} \leqslant n<\tau_{j+1}\right)}{\mathbf{P}(T>n)} \mathbf{P}\left(A_{n}, T>n \mid \tau_{j} \leqslant n<\tau_{j+1}\right) .
$$

Пусть $S_{m}(n)$ - сумма первых $m$ слагаемых последнего ряда, а $R_{m}(n)-$ соответствующий остаток ряда. Тогда

$$
\mathbf{P}\left(A_{n} \mid T>n\right)=S_{m}(n)+R_{m}(n) .
$$

В силу соотношений (3), (39) и (40)

$$
\lim _{n \rightarrow \infty} S_{m}(n)=\frac{c_{1}}{c_{2}} \sum_{j=0}^{m-1} a_{j}
$$

Очевидно, $S_{m}(n) \leqslant 1$ при $n, m \in \mathrm{N}$, поэтому существует конечный предел

$$
\lim _{m \rightarrow \infty} \lim _{n \rightarrow \infty} S_{m}(n)=\frac{c_{1}}{c_{2}} \sum_{j=0}^{\infty} a_{j}
$$


Далее, при $j \in \mathbf{N}_{0}$

$$
\mathbf{P}\left(T>n ; \tau_{j} \leqslant n<\tau_{j+1}\right)=\mathbf{M}\left(\mathbf{P}_{\pi}(T>n) ; \tau_{j} \leqslant n<\tau_{j+1}\right) .
$$

Очевидно, что

$$
\mathbf{P}_{\pi}(T>n)=1-f_{0}\left(f_{1}\left(\ldots f_{n-1}(0) \ldots\right)\right) \leqslant \min _{0 \leqslant i \leqslant n-1}\left(f_{0}^{\prime}(1) \ldots f_{i}^{\prime}(1)\right)=\exp (-M(n))
$$

где $M(n)=\max _{0 \leqslant i \leqslant n}\left(-S_{i}\right)$. Из соотношений $(43),(44)$ следует, что при $j \in \mathbf{N}_{0}$

$$
\mathbf{P}\left(T>n ; \tau_{j} \leqslant n<\tau_{j+1}\right) \leqslant \mathbf{M}\left(\exp (-M(n)) ; \tau_{j} \leqslant n<\tau_{j+1}\right) .
$$

Покажем, что

$$
\lim _{m \rightarrow \infty} \lim _{n \rightarrow \infty} \sum_{j=m}^{\infty} M \exp \left(-M(n) ; \tau_{j} \leqslant n<\tau_{j+1}\right)=0 .
$$

Очевидно, что

$$
\sum_{j=0}^{\infty} \mathbf{M}\left(e^{-M(n)} ; \tau_{j} \leqslant n<\tau_{j+1}\right)=\mathbf{M} e^{-M(n)} .
$$

Известно (см., например, [4]), что при $n \rightarrow \infty$

$$
\mathrm{M} e^{-M(n)} \sim \frac{c_{1}}{\sqrt{n}} \int_{0}^{\infty} e^{-x} d V(x)
$$

где

$$
V(x)=\chi(x)+\sum_{j=1}^{\infty} \mathbf{P}\left(-S_{\tau_{j}} \leqslant x\right),
$$

$\chi(x)$ - индикатор множества $[0, \infty)$. Найдем асимптотику отдельного слагаемого в левой части (47). Из определения строгих нижних лестничных моментов следует, что для произвольного $j \in \mathbf{N}$

$$
\mathbf{M}\left(e^{-M(n)} ; \tau_{j} \leqslant n<\tau_{j+1}\right)=\mathbf{M}\left(e^{S_{\tau_{j}}} ; \tau_{j} \leqslant n<\tau_{j+1}\right) .
$$

Положим при $\lambda \in(0,1)$

$$
\begin{aligned}
\mathbf{M}\left(e^{S_{\tau_{j}}} ; \tau_{j} \leqslant n<\right. & \left.\tau_{j+1}\right) \\
& =\mathbf{M}\left(e^{S_{\tau_{j}}} ; \tau_{j} \leqslant \lambda n, n<\tau_{j+1}\right)+\mathbf{M}\left(e^{S_{\tau_{j}}} ; \lambda n<\tau_{j} \leqslant n<\tau_{j+1}\right) .
\end{aligned}
$$

Поскольку $\exp \left(S_{\tau_{j}}\right) \leqslant 1$, в силу соотношения (4)

$$
\mathbf{M}\left(e^{S_{\tau_{j}}} ; \lambda n<\tau_{j} \leqslant n<\tau_{j+1}\right)=o\left(\frac{1}{\sqrt{n}}\right) .
$$

Далее, используя обозначения леммы 1 , запишем, что

$$
\begin{aligned}
\mathrm{M}\left(e^{S_{\tau_{j}}} ; \tau_{j} \leqslant \lambda n, \tau_{1}^{\prime}>n\right) & \leqslant \mathrm{M}\left(e^{S_{\tau_{j}}} ; \tau_{j} \leqslant \lambda n, n<\tau_{j+1}\right) \\
& \leqslant \mathrm{M}\left(e^{S_{\tau_{j}}} ; \tau_{j} \leqslant \lambda n, \tau_{1}^{\prime}>n(1-\lambda)\right) .
\end{aligned}
$$


Отсюда ввиду независимости $\tau_{1}^{\prime}$ от $\left(S_{\tau_{j}}, \tau_{j}\right)$ и соотношения (3) получаем, что

$$
\begin{aligned}
c_{1} \mathbf{M} e^{S_{\tau_{j}}} & \leqslant \varliminf_{n \rightarrow \infty} \sqrt{n} \mathbf{M}\left(e^{S_{\tau_{j}}} ; \tau_{j} \leqslant \lambda n, n<\tau_{j+1}\right) \\
& \leqslant \varlimsup_{n \rightarrow \infty} \sqrt{n} \mathbf{M}\left(e^{S_{\tau_{j}}} ; \tau_{j} \leqslant \lambda n, n<\tau_{j+1}\right) \\
& \leqslant \frac{c_{1}}{\sqrt{1-\lambda}} \mathbf{M} e^{S_{\tau_{j}}} .
\end{aligned}
$$

Из соотношений (50)-(52) следует, что

$$
\begin{aligned}
c_{1} \mathbf{M} e^{S_{\tau_{j}}} & \leqslant \varliminf_{n \rightarrow \infty} \sqrt{n} \mathbf{M}\left(e^{S_{\tau_{j}}} ; \tau_{j} \leqslant n<\tau_{j+1}\right) \\
& \leqslant \varlimsup_{n \rightarrow \infty} \sqrt{n} \mathbf{M}\left(e^{S_{\tau_{j}}} ; \tau_{j} \leqslant n<\tau_{j+1}\right) \\
& \leqslant \frac{c_{1}}{\sqrt{1-\lambda}} \mathbf{M} e^{S_{\tau_{j}}} .
\end{aligned}
$$

Отсюда, переходя к пределу при $\lambda \rightarrow 0$, получаем, что

$$
\lim _{n \rightarrow \infty} \sqrt{n} \mathbf{M}\left(e^{S_{\tau_{j}}} ; \tau_{j} \leqslant n<\tau_{j+1}\right)=c_{1} \mathbf{M} e^{S_{\tau_{j}}}
$$

Из соотношений (3) (при $j=0$ ), (49), (53) вытекает, что при любом фиксированном $j \in \mathbf{N}_{0}$ и $n \rightarrow \infty$

$$
\mathbf{M}\left(e^{-M(n)} ; \tau_{j} \leqslant n<\tau_{j+1}\right) \sim \frac{c_{1}}{\sqrt{n}} \mathbf{M} e^{S_{\tau_{j}}}
$$

Из соотношений (47), (48) и (54) получаем (46).

Из соотношений (45), (46) находим, что

$$
\lim _{m \rightarrow \infty} \varlimsup_{n \rightarrow \infty} \sqrt{n} \sum_{j=m}^{\infty} \mathbf{P}\left(T>n ; \tau_{j} \leqslant n<\tau_{j+1}\right)=0 .
$$

Отсюда с учетом (39) следует, что

$$
\lim _{m \rightarrow \infty} \varlimsup_{n \rightarrow \infty} R_{m}(n)=0 .
$$

Из соотношений (41) и (42) видно, что

$$
\begin{aligned}
\frac{c_{1}}{c_{4}} \sum_{j=0}^{\infty} a_{j} & \leqslant \varliminf_{n \rightarrow \infty} \mathbf{P}\left(A_{n} \mid T>n\right) \leqslant \varlimsup_{n \rightarrow \infty} \mathbf{P}\left(A_{n} \mid T>n\right) \\
& \leqslant \frac{c_{1}}{c_{4}} \sum_{j=0}^{\infty} a_{j}+\lim _{m \rightarrow \infty} \varlimsup_{n \rightarrow \infty} R_{m}(n),
\end{aligned}
$$

Отсюда, учитывая (55), получаем утверждение леммы 10.

Лемма 11. В условиях теоремъ 1 при $n \rightarrow \infty$

$$
\left\{\xi_{n} / m_{n} \mid T>n\right\} \stackrel{\mathscr{D}}{\longrightarrow} \mu,
$$

где $\mu$ - некоторая неотрицательная случайная величина, для которой $\mathbf{P}(\mu>0)>0$. 
Доказательство. Пусть $x$ - точка непрерывности сразу всех функций распределения случайных величин $\mu^{(j)}, j \in \mathbf{N}_{0}$, задействованных в лемме 9. Очевидно, что множество $D$ таких точек всюду плотно на числовой прямой. Ясно, что если $x \in D \cap(0, \infty)$, то при каждом $j \in \mathbf{N}_{0}$

$$
\mathbf{P}\left(\frac{\xi_{n}}{m_{n}}>x, T>n \mid \tau_{j} \leqslant n<\tau_{j+1}\right)=\mathbf{P}\left(\frac{\xi_{n}}{m_{n}}>x \mid \tau_{j} \leqslant n<\tau_{j+1}\right),
$$

и поэтому ввиду леммы 9

$$
\lim _{n \rightarrow \infty} \mathbf{P}\left(\frac{\xi_{n}}{m_{n}}>x, T>n \mid \tau_{j} \leqslant n<\tau_{j+1}\right)=\mathbf{P}\left(\mu^{(j)}>x\right),
$$

причем $\mathbf{P}\left(\mu^{(0)}>x\right)>0$ при достаточно малых $x$. Применяя лемму 10 , получаем, что при любом фиксированном $x \in D \cap(0, \infty)$

$$
\lim _{n \rightarrow \infty} \mathbf{P}\left(\frac{\xi_{n}}{m_{n}}>x \mid T>n\right)=\frac{c_{1}}{c_{2}} \sum_{j=0}^{\infty} \mathbf{P}\left(\mu^{(j)}>x\right)<\infty
$$

где сумма ряда больше 0 при достаточно малых $x$. Если же $x \leqslant 0$, то

$$
\lim _{n \rightarrow \infty} \mathbf{P}\left(\frac{\xi_{n}}{m_{n}}>x \mid T>n\right)=1
$$

Из соотношения (57) ввиду монотонности $\mathbf{P}\left(\mu^{(j)}>x\right)$ по $x$ следует, что

$$
\lim _{x \rightarrow \infty} \frac{c_{1}}{c_{2}} \sum_{j=0}^{\infty} \mathbf{P}\left(\mu^{(j)}>x\right)=0 .
$$

Из соотношений (57)-(59) вытекает утверждение леммы 11.

Лемма 12. В условиях теоремы 1 при каждом фиксированном $u \in(0,1)$

$$
\left\{\sup _{t \in[u, 1]}\left|X_{n}(t)-X_{n}(u)\right| \mid T>n\right\} \stackrel{\mathscr{D}}{\longrightarrow} 0 .
$$

Доказательство. Ввиду леммы 10 достаточно показать, что при любых фиксированных $\varepsilon \in(0, \infty), j \in \mathbf{N}_{0}$

$$
\lim _{n \rightarrow \infty} \mathbf{P}\left(\sup _{t \in[u, 1]}\left|X_{n}(t)-X_{n}(u)\right| \geqslant \varepsilon \mid \tau_{j} \leqslant n<\tau_{j+1}\right)=0,
$$

что вследствие соотношения (4) эквивалентно равенству

$$
\lim _{n \rightarrow \infty} \mathbf{P}\left(\sup _{t \in[u, 1]}\left|X_{n}(t)-X_{n}(u)\right| \geqslant \varepsilon \mid \tau_{j} \leqslant \lambda n, n<\tau_{j+1}\right)=0,
$$

где $\lambda=u / 2$. Поскольку случайная последовательность $\left\{\xi_{n} / m_{n}\right\}$, рассматриваемая при фиксированной случайной среде, является мартингалом, в силу неравенства Дуба

$$
\mathbf{P}_{\pi}\left(\sup _{t \in[u, 1]}\left|X_{n}(t)-X_{n}(u)\right| \geqslant \varepsilon\right) \leqslant \frac{1}{\varepsilon^{2}} \mathbf{M}_{\pi}\left(X_{n}(1)-X_{n}(u)\right)^{2}
$$


Отсюда, учитывая лемму 7 , получаем, что

$$
\begin{aligned}
\mathbf{P}\left(\sup _{t \in[u, 1]} \mid X_{n}(t)\right. & \left.-X_{n}(u)|\geqslant \varepsilon| \tau_{j} \leqslant \lambda n, n<\tau_{j+1}\right) \\
& =\mathbf{M}\left(\mathbf{P}_{\pi}\left(\sup _{t \in[u, 1]}\left|X_{n}(t)-X_{n}(u)\right| \geqslant \varepsilon\right) \mid \tau_{j} \leqslant \lambda n, n<\tau_{j+1}\right) \\
& \leqslant \frac{1}{\varepsilon^{2}} \mathbf{M}\left(\mathbf{M}_{\pi}\left(X_{n}(1)-X_{n}(u)\right)^{2} \mid \tau_{j} \leqslant \lambda n, n<\tau_{j+1}\right) \\
& =\frac{1}{\varepsilon^{2}} \mathbf{M}\left(2\left(b_{n}-b_{[n u]}\right)+a_{n}-a_{[n u]} \mid \tau_{j} \leqslant \lambda n, n<\tau_{j+1}\right) .
\end{aligned}
$$

Применяя теперь лемму 3, приходим к соотношению (60). Лемма 12 доказана.

Доказательство теоремы 1. Ввиду леммы 11 при $n \rightarrow \infty$

$$
\left\{X_{n}(1) \mid T>n\right\} \stackrel{\mathscr{D}}{\longrightarrow} \mu,
$$

откуда, применяя лемму 12 , получаем, что при каждом фиксированном $u \in(0,1)$ и $n \rightarrow \infty$

$$
\left\{X_{n}(t), t \in[u, 1] \mid T>n\right\} \Rightarrow \mu
$$

(здесь $\mu$ означает случайный процесс, сопоставляющий каждому $t \in(0,1]$ случайную величину, стоящую в правой части (61)). Осталось показать, что

$$
\lim _{\delta \downarrow 0} \varlimsup_{n \rightarrow \infty} \mathbf{P}\left(w_{X_{n}}(\delta, u, 1) \geqslant \varepsilon \mid T>n\right)=0
$$

где $w_{f}(\delta, u, v)$ означает модуль непрерывности функции $f \in D[u, v], 0 \leqslant u<v<\infty$, то есть

$$
w_{f}(\delta, u, v)=\sup |f(s)-f(t)|
$$

где супремум берется по всем $s, t$ таким, что $s, t \in[u, v],|t-s|<\delta, \delta \in(0, \infty)$. Заметим, что при любых $u \in(0,1)$ и $\varepsilon \in(0, \infty)$

$$
\left\{w_{X_{n}}(\delta, u, 1) \geqslant \varepsilon\right\} \subset\left\{\sup _{t \in[u, 1]}\left|X_{n}(t)-X_{n}(u)\right| \geqslant \frac{\varepsilon}{2}\right\}
$$

Отсюда, снова применяя лемму 12 , получаем соотношение (63). Из соотношений (62) и (63) следует (см. параграф 15 в [7]) утверждение теоремы 1. 


\section{Список литературы}

1. Афанасьев В. И., Новая предельная теорема для критического ветвящегося процесса в случайной среде. Дискретная математика (1997) 9, №3, 52-67.

2. Афанасьев В. И., Предельные теоремы для умеренно докритического ветвящегося процесса в случайной среде. Дискретная математиха (1998) 10, №1, 141-157.

3. Афанасьев В. И., Предельные теоремы для промежуточно докритического и строго докритического ветвящихся процессов в случайной среде. Дискретная математика (2001) 13, №1, 132-157.

4. Козлов М. В., Об асимптотике вероятности невырождения критических ветвящихся процессов в случайной среде. Теория вероятностей и ее применения (1976) 21, №4, 813-825.

5. Афанасьев В. И., Предельная теорема для критического ветвящегося процесса в случайной среде. Дискретная математиха (1993) 5, №1, 45-58.

6. Borovkov K. A., Vatutin V. A., Reduced critical branching processes in random environment. Stoch. Proc. Appl. (1997) 71, 225-240.

7. Биллингсли П., Сходимость вероятностных мер. Наука, Москва, 1977.

8. Geiger J., Kersting G., The survival probability of a critical branching process in random environment. Теория вероятностей и ее применения (2000) 45, N3, 607-615.

Статья поступила 10.11.2001 